\title{
BMJ Open Comparison of effects of different dietary interventions on cognitive function in Alzheimer's disease: protocol for systematic review and network meta-analysis
}

\author{
Lili Chen (D) , ${ }^{1,2}$ Xinhua Xu (D) , ${ }^{2}$ Huizhen Cao, ${ }^{2}$ Hong Li ${ }^{1,2}$
}

To cite: Chen $\mathrm{L}, \mathrm{Xu} X, \mathrm{CaO} \mathrm{H}$, et al. Comparison of effects of different dietary interventions on cognitive function in Alzheimer's disease: protocol for systematic review and network meta-analysis. BMJ Open 2021;11:e042997. doi:10.1136/ bmjopen-2020-042997

- Prepublication history for this paper is available online. To view these files, please visit the journal online (http://dx.doi org/10.1136/bmjopen-2020042997).

Received 21 July 2020 Revised 26 November 2020 Accepted 08 December 2020

D Check for updates

(c) Author(s) (or their employer(s)) 2021. Re-use permitted under CC BY-NC. No commercial re-use. See rights and permissions. Published by BMJ.

${ }^{1}$ Sheng Li Clinical Medical College of Fujian Medical University, Fujian Provincial Hospital, Fuzhou, Fujian, China ${ }^{2}$ School of Nursing, Fujian Medical University, Fuzhou, Fujian, China

\section{Correspondence to}

Professor Lili Chen;

fjslyy2018@outlook.com and

Hong Li;

leehong99@126.com

\section{ABSTRACT}

Introduction Alzheimer's disease (AD) is the most common neurodegenerative disease and is characterised by cognitive impairment. Non-pharmacological treatments such as diet therapy have been widely investigated in studies on AD. Given the synergistic effects of nutrients present in foods, considering overall dietary composition rather than focusing on a single nutrient may be more useful for evaluating the relationship between diet and $A D$ cognition. The present study aimed to assess the efficacy of different dietary interventions (eg, ketogenic and Mediterranean diets) on cognitive function in patients with $A D$ in a systematic review and pairwise and network metaanalyses of randomised controlled trials or clinical trials. Methods and analysis Two reviewers will independently conduct searches of PubMed, Cochrane Central Register of Controlled Trials, Embase, CINAHL, PsycINF0 and China National Knowledge Infrastructure databases. Data will be extracted from selected studies and risk of bias will be assessed using the revised Cochrane risk-of-bias tool, and evidence quality will be assessed according to the Grading of Recommendations, Assessment, Development and Evaluation framework. The primary outcome of interest is cognitive function in patients with $\mathrm{AD}$; secondary outcomes include biochemical biomarkers of $A D$ and oxidative stress and/or inflammatory biomarkers in cerebrospinal fluid or plasma. For each outcome, random-effects pairwise and network meta-analyses will be carried out to determine the pooled relative effect of each intervention relative to every other intervention.

Ethics and dissemination As this study is based solely on published literature, no ethics approval is required. The research will be published in a peer-reviewed journal.

\section{BACKGROUND}

Alzheimer's disease (AD) is the most common neurodegenerative disease and is characterised by cognitive impairment. The risk of $\mathrm{AD}$ increases with factors such as age and treatment with ineffective drugs. Over the past few decades, non- pharmacological treatment strategies such as diet therapy have been investigated in studies on $\mathrm{AD} .^{1{ }^{2}}$
Strengths and limitations of this study

- The protocol will attempt to answer the important question of which dietary interventions have the most benefit for the cognitive function of patients with Alzheimer's disease

- The study will be conducted in strict accordance with the recommendations of the Cochrane Handbook for Systematic Reviews of Interventions.

- We expect that the pooled effect will be influenced by the high statistical heterogeneity and quality of analysed trials.

Specific dietary nutrients such as vitamins B6 and B12, omega-3 polyunsaturated fatty acids (PUFAs), vitamin $\mathrm{D}$, folate, and antioxidant vitamins $\mathrm{A}, \mathrm{C}$ and $\mathrm{E}$ have been linked to $\mathrm{a}$ reduced risk of dementia in observational studies, ${ }^{34}$ although it has not been possible to draw definitive conclusions regarding the effects of specific types of nutritional supplementation on cognitive function. Additionally, regular intake of fish, vegetables, fruits and nuts is associated with a reduced risk of cognitive impairment in $\mathrm{AD},{ }^{56}$ and the Mediterranean diet (MeDi), Dietary Approaches to Stop Hypertension (DASH) and hybrid Mediterranean-DASH Intervention for Neurodegenerative Delay (MIND) have shown cognitive benefits for patients with $\mathrm{AD}$ that are attributable to their inclusion of fruit and fish, vegetables, unsaturated fats and whole-grain products. ${ }^{7-11}$

Given the synergistic effects of nutrients present in foods, considering overall dietary composition rather than single nutrients may be a more reasonable approach to evaluating the relationship between diet and $\mathrm{AD}$ cognition. ${ }^{4}$ However, although some systematic reviews and pairwise meta-analyses have been published regarding the effects of the MeDi, 
ketogenic diet $(\mathrm{KD})$ and $\mathrm{DASH}$ on $\mathrm{AD}$, it is unclear which type of diet is the most effective for improving cognitive function in patients with $\mathrm{AD}$ as there have been no systematic reviews or network meta-analyses to date comparing different dietary interventions in the management of AD.

To address this issue, we will investigate the effects of different dietary interventions on cognitive function in patients with $\mathrm{AD}$ by performing a systematic review and conducting pairwise and network meta-analyses of published randomised controlled trials (RCTs) and clinical trials.

\section{METHODS AND DESIGN}

We devised a protocol based on Preferred Reporting Items for Systematic Reviews and Meta-Analyses ${ }^{12}$ guidelines that was registered in the International Prospective Register of Systematic Reviews (CRD42020177298) and followed the Network Meta-Analysis Protocol Guidelines (Cochrane Handbook for Systematic Reviews of Interventions). ${ }^{13}$

\section{Inclusion and exclusion criteria}

Studies that meet the following criteria will be included in the analysis.

\section{Participants}

We will include any research that used their country's standard $\mathrm{AD}$ diagnostic criteria and neuropsychological tests to diagnose AD. Taking China as an example, we will use the National Institute of Neurological and Communicative Disorders and Stroke-Alzheimer Disease and Related Disorders Association criteria, and/or National Institute on Aging (NIA) and the Alzheimer's Association criteria, and/or the International Working Group, Second Edition and/or the Diagnostic and Statistical Manual of Mental Disorders, Third or Fourth Edition, respectively). ${ }^{14}$ We will use the Mini-Mental State Examination (MMSE)/Montreal Cognitive Assessment/Clinical Dementia Rating Scale for additional screening. We will consider patients with $\mathrm{AD}$ aged $>60$ years old, without restrictions regarding sex, race/ethnicity, geography, dwelling or chronic diseases.

\section{Dietary interventions}

We will include all dosages or types of dietary intervention with no restrictions on the duration of the intervention or whether they were combined with drugs. However, selected studies will include at least one of the following interventional diets and one control group (indirect evidence) (eg, MeDi and the NIA-recommended diet) or at least two interventional diets (direct evidence). Eligible dietary interventions are as follows:

- MeDi. This natural diet consumed by inhabitants of the Mediterranean coast is reported to prevent $\mathrm{AD}$ and cognitive decline. ${ }^{15-20}$ The diet consists of a high intake of vegetables, fruit, legumes, olive oil, fish, grains and nuts, and moderate consumption of red wine. Because of these components, the MeDi is rich in phenolic compounds, omega-3 PUFAs and vitamins that promote cerebrovascular health and reduce oxidative stress and chronic inflammation. ${ }^{15}$

- DASH. This diet includes a high intake of fruit and vegetables, low-fat dairy, and whole grains. Long-term (6 years) adherence to DASH was shown to enhance overall cognition and verbal memory in older adults, and high DASH compliance was associated with consistently high cognitive functioning in patients with AD. $21^{21}$

- KD. This diet is very high in fat and low in carbohydrates. There is considerable interest in the potential value of ketone-inducing therapies for the treatment of $\mathrm{AD}$ (neuroketotherapeutics (NKT)) ${ }^{22}$; they are thought to alleviate physical AD symptoms through bioenergy recovery, direct and indirect regulations of antioxidant and inflammatory pathways, or both. Preliminary evidence suggests that NKT is a necessary remission therapy for $\mathrm{AD}{ }^{22-24}$

- MIND. This diet emphasises the intake of berries and dark green leafy vegetables, which are natural sources of vitamin K, folic acid, lutein, lycopene, beta-cyclin, and alpha-carotene and beta-carotene. ${ }^{25}$

- Nordic diet. This diet, which is consumed in Scandinavian countries and is similar to the MeDi, includes large amounts of fruits and vegetables and is rich in fish omega-3 PUFAs, phenolic compounds and wholegrain products. An observational study concluded that adherence to the Nordic diet was associated with better cognitive performance. ${ }^{11}$

- Calorie restriction (CR). Also known as dietary restriction, CR refers to a dietary regimen low in calories without undernutrition, which may influence agerelated changes in the brain and ultimately enhance health and extend life span. It was reported that the incidence of $\mathrm{AD}$ was lower among individuals who habitually consumed fewer calories. ${ }^{6}$ 26-28

- Modified Mediterranean-KD, Multicultural Healthy Diet, Mediterranean-style KD, American Heart Association Diet, low fat-diet, energy-restricted diets, isocaloric diets, diabetes prevention-type diets, standard dietary recommendations or ad libitum diets will be considered (see the Search strategy section for details).

\section{Outcome measures}

The primary outcome of interest is cognitive function. Cognition will be assessed using the MMSE, and/or Alzheimer's Disease Assessment Scale cognitive subscale, and/or Montreal Cognitive Assessment, and/or Clock Drawing Test, and/or Test Your Memory, and/or Loewenstein Occupational Therapy Cognitive Assessment, and/ or Neurobehavioral Cognitive Status Examination. The specific cognition domains assessed according to DSM, Fifth Edition, criteria include complex attention, executive function, learning and memory, language, perceptual motor and social cognition. Common assessment scales include the Stroop test, and/or Categorical Verbal Fluency 
test, and/or Clinical Memory Scale, and/or Wechsler Memory Scale, and/or Boston Diagnostic Aphasia Examination, and/or Rey Complex Figure Test. Secondary outcome measures are biochemical biomarkers and oxidative stress and/or inflammatory biomarkers in the cerebrospinal fluid or plasma of patients with AD. Effect sizes of continuous and dichotomous variables will be determined as the standard mean difference (SMD) and OR, respectively.

\section{Types of study}

Study design will include RCTs and clinical trials.

The following types of RCTs and clinical trials will be excluded.

- Enrolled healthy participants or patients with mild cognitive decline or other types of non-Alzheimer dementia; includes cases of familial $\mathrm{AD}$ emerging before the age of 50 years or $\mathrm{AD}$ related to other genetic diseases (eg, trisomy of chromosome 21); there were other interventions that could affect cognitive function; or patients had other cognitive comorbidities or mental illnesses.

- Other interventions such as exercise interventions and cognitive therapy were used. For multimodal studies (eg, the Finnish Geriatric Intervention Study to Prevent Cognitive Impairment and Disability), we will include the dietary component (if available).

- Intervention studies based on dietary supplements (eg, vitamins B or E, and omega-3 PUFAs) or multicomponent supplements (eg, Souvenaid).

- Studies with exercise/medication cointervention that was not applied to all intervention/control groups.

- Examined plasma nutrient levels, nutritional status or food intake without associating these directly to $\mathrm{AD}$ status or progression.

- Descriptive studies, reviews and case studies; nonhuman animal model studies (in vivo or in vitro); and studies with full texts published in languages other than English or Chinese.

\section{Search strategy}

Two reviewers (XHX and HZC) will independently perform the search, and any differences will be resolved by discussion or by a third reviewer (LLC). We will conduct searches in PubMed, Embase, Cochrane Library, CINAHL, PsycINFO, Google Scholar and China National Knowledge Infrastructure databases up to November 2020. Only English/Chinese studies will be included, with no restrictions on publication status or publication year.

We will use the following terms to search for articles (eg, in the PubMed database).

1. Alzheimer disease (Medical Subject Heading (MeSH) terms).

2. Dementia, Senile OR Senile Dementia OR Dementia, Alzheimer Type OR Alzheimer Type Dementia OR Alzheimer-Type Dementia (ATD) OR Alzheimer Type Dementia (ATD) OR Dementia, AlzheimerType (ATD) OR Alzheimer Type Senile Dementia OR
Primary Senile Degenerative Dementia OR Dementia, Primary Senile Degenerative OR Alzheimer Sclerosis OR Sclerosis, Alzheimer OR Alzheimer Syndrome OR Alzheimer Dementia OR Dementia, Alzheimer OR Senile Dementia, Alzheimer Type OR Acute Confusional Senile Dementia OR Senile Dementia, Acute Confusional OR Dementia, Presenile OR Presenile Dementia OR Alzheimer Disease, LateOnset OR Alzheimer's Disease, Focal Onset OR Familial Alzheimer Disease (FAD) OR Alzheimer Disease, Familial (FAD) OR Alzheimer Disease, Early Onset OR Presenile Alzheimer Dementia.

3. \#1 or \#2.

4. Diet (MeSH terms) OR Diet, Western (MeSH terms) OR Diet, Palaeolithic (MeSH terms) OR Diet, HighFat (MeSH terms) OR Diet, Carbohydrate-Restricted (MeSH terms) OR Diet, Ketogenic (MeSH terms) OR Diet, Mediterranean (MeSH terms) OR Diet, ProteinRestricted (MeSH terms) OR Diet, Fat-Restricted (MeSH terms) OR Diet, Macrobiotic (MeSH terms) OR Diet, Vegetarian (MeSH terms) OR Diet, SodiumRestricted (MeSH terms) OR Diet, Reducing (MeSH terms) OR Diet, Cariogenic (MeSH terms) OR Diet, Atherogenic (MeSH terms) OR Diet Therapy (MeSH terms) OR Diet Surveys (MeSH terms) OR Diet Fads (MeSH terms) OR Diet, Diabetic (MeSH terms) OR Diet, High-Protein Low-Carbohydrate (MeSH terms) OR Diet, High-Protein (MeSH terms) OR Diet, Carbohydrate Loading (MeSH terms) OR Diet, Vegan (MeSH terms) OR Diet, Food, and Nutrition (MeSH terms) OR Artificially Sweetened Beverages (MeSH terms) OR Food, Formulated (MeSH terms) OR Feeding Behaviour (MeSH terms) OR Caloric Restriction (MeSH terms) OR Dietary Approaches To Stop Hypertension (MeSH terms) OR protein, Drosophila (MeSH terms) OR Diet, Gluten-Free (MeSH terms).

5. Mediterranean diet OR MD diet OR MeDi diet OR DASH OR dietary approaches to stop Hypertension OR the Mediterranean DASH diet Intervention for Neurological Delay (MIND) diet OR MIND diet OR Ketogenic diet OR medium chain triglyceride diet OR MCT diet OR modified Atkins diet OR MAD diet OR Atkins OR Calorie Restriction (CR) OR low carbohydrate OR high carbohydrate OR low fat OR high fat OR low protein OR high protein OR vegetarian OR vegan OR low glycaemic index OR low glycaemic load OR Palaeolithic OR low-calorie OR Atkins OR low sodium OR Modified Mediterraneanketogenic diet (MMK) OR Multicultural Healthy Diet (MHD) diet OR Mediterranean-style ketogenic diet (MMKD) OR The American Heart Association Diet (AHAD) OR low fat diet OR energy-restricted diets OR isocaloric diet OR diabetes prevention-type diets OR standard dietary recommendations OR ad libitum diets.

6. \#4 or \#5. 
7. Cognition (MeSH terms) OR Cognition Disorders (MeSH terms) OR Mental Status and Dementia Tests (MeSH terms).

8. Cognitions OR Cognitive Function OR Cognitive Functions OR Function, Cognitive OR Functions, Cognitive.

9. \#7 or \#8.

10. random* or clinical*, not animals.

11. (\#3 and \#6 and \#9 and \#10).

We will also verify the reference list of retrieved articles, systematic reviews and meta-analyses for relevant studies, and search other resources (eg, Google Scholar, ClinicalTrials.gov and Open Grey). For studies not reported in Chinese or English, we will contact the author to determine whether an English version is available.

\section{Study selection process}

Two reviewers (XHX and HZC) will independently select the studies based on the inclusion criteria. Before study selection, all of the retrieved study citations will be imported into Covidence and duplicates will be removed. Before formal screening of titles and abstracts, we will conduct an intratester agreement (kappa) test between the two reviewers, who will select 50 citations using a random number table until they reach an agreement of $80 \%$. The reviewers will then read the titles and abstracts of retrieved studies, remove those that do not qualify and further screen potentially qualifying studies based on the pre-established inclusion and exclusion criteria. If the information in the study is insufficient, we will contact the author for more information. Any disagreement between the two reviewers will be adjudicated by a third investigator (LLC). The study selection process and reasons for study exclusion will be outlined in a flowchart.

\section{Data extraction}

Data will be independently extracted by two reviewers (XHX and HZC) when they have reached a consensus, with the input of a third investigator (LLC) in the event of a disagreement. If the study data are essential but unclear, we will contact the author for further details. The following data will be extracted from each study.

1. Study information (name of the first author, publication year, country of origin, title and study design).

2. Patient characteristics (sample size, average age, number of women/men, race/ethnicity, $\mathrm{AD}$ diagnostic criteria, $\mathrm{AD}$ severity, mean baseline cognitive function, medication and apolipoprotein (APOE4 status).

3. Intervention and control (dietary protocols, duration of treatment, number of treatment arms, specification of the control group (if available), dropouts and funding source).

4. Methodological information (description of randomisation, sequence concealment, blinding, incomplete outcome data, and selective reporting and other potential sources of bias).

5. Outcomes (primary and secondary outcomes and measurement dates).
Risk of bias assessment

The two reviewers (XHX and HZC) will independently assess the risk of bias in the included studies using the Cochrane Collaboration's tool. ${ }^{29}$ Any disagreement will be resolved through discussion by the two reviewers or decided by a third investigator (LLC). The domains for risk of bias are as follows:

1. Selection bias (randomised sequence generation and allocation concealment).

2. Performance bias (blinding of participants and personnel).

3. Detection bias (blinding of outcome assessment).

4. Attrition bias (incomplete outcome data).

5. Reporting bias (selective reporting).

6. Other biases (including baseline imbalance, claim of data fraudulence, differential diagnostic activity and contamination).

For each domain, we will categorise the risk of bias as low, unclear or high according to the Cochrane Handbook for Systematic Reviews of Interventions.

\section{Data synthesis}

\section{Description of available data}

We will derive each pairwise comparison from descriptive statistics of available data and selected variables for study characteristics, patient characteristics, interventions and outcomes measures (eg, age, sex, study length and outcome-relevant baseline risk factors). We will evaluate interventions to identify specific nodes that will be used in the network meta-analysis. If the network is connected, a random-effects network meta-analysis will be carried out. ${ }^{30-33}$ A network diagram will be used for outcomes to present direct comparisons between different dietary interventions and corresponding control groups. ${ }^{2} \mathrm{We}$ will also analyse the contribution matrix to identify direct comparisons that have a more significant influence on the network's relative effects. ${ }^{34}$

\section{Pairwise and network meta-analyses}

We will initially compare all interventions with available direct evidence in separate pairwise meta-analyses. We will use the SMD as the effect measure for continuous outcome variables. Heterogeneity across studies will be assessed with the $Q$ test and the $\mathrm{I}^{2}$ statistic, with substantial heterogeneity defined as $\mathrm{I}^{2}>50 \%$. The effects will be pooled with a random-effects model, accounting for potential between-study heterogeneity. Study-specific effect sizes, along with 95\% CIs, will be displayed in forest plots.

We will then pool all studies using a network metaanalysis model within a Bayesian framework. As an extension of standard pairwise meta-analysis models, network meta-analysis allows for simultaneous comparison of multiple interventions while preserving the internal randomisation of individual trials. Using a randomeffects network meta-analysis for the outcome, we will estimate all possible pairwise relative effects and perform clinically meaningful ranking of the different dietary 
interventions. ${ }^{35}$ For each outcome, we will use the distribution of ranking probabilities and surface under the cumulative ranking curve to estimate the relative rank of the different diets. ${ }^{36}$ We will also estimate relative efficacy and safety rankings of the treatments, which will be presented as rank plots. For multiarm trials, we will account for the correlation of effect sizes. Summary mean differences will be presented in a league table.

All data will be analysed using STATA V.13 and WinBUGS V.1.4.3. ${ }^{37}$ Results will be reported as SMD, along with $95 \%$ CIs based on 100000 Monte Carlo simulations and vague priors. Model convergence will be assessed by examining trace and history plots and by calculating the GelmanRubin statistic. Forest plots and other data will be analysed using appropriate packages in STATA V.13.

If network meta-analysis is not feasible, pairwise metaanalysis will be conducted for interventions with direct evidence only, and the results will be presented in the form of forest plots. If there is too little research or the heterogeneity is too great across studies (especially when apparent clinical or methodological heterogeneity precludes a meta-analysis), a system description will instead be provided.

\section{Assumption of transitivity}

We will evaluate the transitivity assumption by comparing the distribution of clinical and methodological variables that are considered as potential effect modifiers (eg, changes in AD severity, medication, APOE4 status, mean baseline cognitive function and age) across dietary interventions. $^{38} 39$

\section{Assessment of inconsistency}

Statistical inconsistency-that is, disagreement between different sources of evidence-can be evaluated using local and global approaches. ${ }^{40}$ We will first use the designby-treatment interaction model and $\mathrm{I}^{2}$ statistic to identify global inconsistencies from all possible sources in the network. ${ }^{4142}$ If an inconsistency is detected, we will explore local inconsistencies using the loop-specific approach, ${ }^{43}$ as well as the node-splitting approach, ${ }^{44}$ which identifies comparisons in which direct estimates disagree with indirect evidence from the entire network.

\section{Subgroup and sensitivity analyses}

We will explore the possible sources of significant heterogeneity or inconsistency by subgroup and metaregression analyses. The former is planned for genes (eg, APOE4), age, $\mathrm{AD}$ severity, region, education level, comorbidities and drug use. We will quantify effects by metaregression analysis by adding covariates to the network meta-analysis models if appropriate.

We will perform sensitivity analyses to assess the robustness of results on primary outcomes according to sample size (excluding small studies with sample size of $<50$ ) and study quality (excluding studies with high risk in $\geq 1$ domain or with unclear risk in $\geq 3$ domains as determined using the Cochrane Collaboration's tool for assessing risk of bias).$^{45}$ We will remove each study from the metaanalysis one at a time and recalculate the summary effect. A study will be considered influential if its removal changes the magnitude of the pooled effect by $>10 \% .{ }^{46}$

\section{Small-study effects and publication bias}

We will use a comparison-adjusted funnel plot to assess the presence of small-study effects. ${ }^{2}$ If the meta-analysis comprises $\geq 8$ studies, we will use a funnel plot to evaluate publication bias, and its asymmetry will be evaluated with Egger's test.

\section{Quality of evidence}

Taking into account the specific requirements of nutrition research, NutriGrade tools have been specifically developed to assess the association between or effects of different nutritional factors and outcomes in metaanalyses. The NutriGrade scoring system evaluates the following items: (1) risk of bias, study quality, and study limitations; (2) precision; (3) heterogeneity; (4) directness; (5) publication bias; (6) funding bias; (7) study design; (8) effect size; and (9) dose-response. In a previous meta-analysis of RCTs, the NutriGrade score varied between 2.9 (very low) and 8.8 (high). ${ }^{47}$ The Grading of Recommendations, Assessment, Development and Evaluation approach will be used to assess the quality of clinical trials.

\section{Patient and public involvement}

There will be no patients involved in this study.

\section{DISCUSSION}

Nutrition is an essential and modifiable risk factor that has an important role in strategies to prevent or delay the onset of dementia. Given the increasing prevalence and incidence of $\mathrm{AD}$ and the potential impact of diet on cognitive function in patients with $\mathrm{AD}$, the present systematic review with network meta-analysis is highly relevant from clinical and practical perspectives. The results are expected to guide evidence-based decision-making in $\mathrm{AD}$ prevention strategies and support reliable recommendations for $\mathrm{AD}$ management.

\section{ETHICS AND DISSEMINATION}

Ethical approval will not be required because this study is based solely on published literature that meets accepted ethical standards, and there will be no concerns about privacy. With the aim of disseminating the evidence obtained, the results will be published in a peer-reviewed international journal to improve clinical practices with scientific evidence.

Correction notice This article has been corrected since it first published. The provenance and peer review statement has been included.

Acknowledgements The authors thank Ying Chen for useful suggestions on our protocol, Bixia Wang for searching for the studies, and Xinli Ji and Ping Zhang for formatting the manuscript. 
Contributors Lili Chen and Xinhua Xu are the co-first authors and contributed equally to the protocol. Lili Chen provided study conception and funding support. Xinhua $\mathrm{Xu}$ assisted with the design, drafting the protocol and registered the protocol in the PROSPERO database. Huizhen Cao provided input on the design and drafting of the protocol. Lili Chen and Hong Li are the co- corresponding authors. Hong Li helped to provide study conception and funding support. All authors read and approved the final protocol.

Funding LLC is funded by high-level hospital foster grants from Fujian Provincial Hospital, Fujian Province, China (no. 2019HSJJ12) and Fujian Provincial Medical Innovation Project (no. 2019J01501).

Disclaimer This article was edited for proper English language, grammar, punctuation, spelling and overall style by one or more of the highly qualified native English-speaking editors at Charlesworth Author Services.

\section{Competing interests None declared.}

Patient and public involvement Patients and the public were not involved in the design, conduct, reporting or dissemination plans of this research.

Patient consent for publication Not required.

Provenance and peer review Not commissioned; externally peer reviewed.

Data availability statement № additional data are available.

Open access This is an open access article distributed in accordance with the Creative Commons Attribution Non Commercial (CC BY-NC 4.0) license, which permits others to distribute, remix, adapt, build upon this work non-commercially, and license their derivative works on different terms, provided the original work is properly cited, appropriate credit is given, any changes made indicated, and the use is non-commercial. See: http://creativecommons.org/licenses/by-nc/4.0/.

\section{ORCID iDs}

Lili Chen http://orcid.org/0000-0003-0612-9422

Xinhua Xu http://orcid.org/0000-0002-2288-0008

\section{REFERENCES}

1 Pistollato F, Iglesias RC, Ruiz R, et al. Nutritional patterns associated with the maintenance of neurocognitive functions and the risk of dementia and Alzheimer's disease: A focus on human studies. Pharmacol Res 2018;131:32-43.

2 Chaimani A, Higgins JPT, Mavridis D, et al. Graphical tools for network meta-analysis in STATA. PLoS One 2013;8:e76654.

3 Coley N, Vaurs C, Andrieu S. Nutrition and cognition in aging adults. Clin Geriatr Med 2015;31:453-64.

4 Rosenberg A, Mangialasche F, Ngandu T, et al. Multidomain interventions to prevent cognitive impairment, Alzheimer's disease, and dementia: from finger to world-wide fingers. J Prev Alzheimers Dis 2020;7:29-36.

5 van den Brink AC, Brouwer-Brolsma EM, Berendsen AAM, et al. The Mediterranean, Dietary Approaches to Stop Hypertension (DASH), and Mediterranean-DASH Intervention for Neurodegenerative Delay (MIND) Diets Are Associated with Less Cognitive Decline and a Lower Risk of Alzheimer's Disease-A Review. Adv Nutr 2019;10:1040-65.

6 Muñoz Fernández SS, Ivanauskas T, Lima Ribeiro SM. Nutritional strategies in the management of Alzheimer disease: systematic review with network meta-analysis. J Am Med Dir Assoc 2017;18:897.e13-897.

7 Petersson SD, Philippou E. Mediterranean diet, cognitive function, and dementia: a systematic review of the evidence. Adv Nutr 2016;7:889-904.

8 Lourida I, Soni M, Thompson-Coon J, et al. Mediterranean diet, cognitive function, and dementia: a systematic review. Epidemiology 2013;24:479-89.

9 Morris MC, Tangney CC, Wang Y, et al. Mind diet associated with reduced incidence of Alzheimer's disease. Alzheimers Dement 2015;11:1007-14.

10 Martínez-Lapiscina EH, Clavero P, Toledo E, et al. Mediterranean diet improves cognition: the PREDIMED-NAVARRA randomised trial. $J$ Neurol Neurosurg Psychiatry 2013;84:1318-25.

11 Männikkö R, Komulainen P, Schwab U, et al. The Nordic diet and cognition--The DR's EXTRA Study. Br J Nutr 2015;114:231-9.

12 Swartz MK. The PRISMA statement: a guideline for systematic reviews and meta-analyses. J Pediatr Health Care 2011;25:1-2.

13 Moher D, Shamseer L, Clarke M, et al. Preferred reporting items for systematic review and meta-analysis protocols (PRISMA-P) 2015 statement. Syst Rev 2015;4:1.
142018 China Guidelines for Diagnosis and Treatment of Dementia and Cognitive Impairment (2): Guidelines for Diagnosis and Treatment of Alzheimer's Disease[J](in Cinese). National Medical Journal of China 2018;98:965-70.

15 Omar SH. Mediterranean and mind diets containing olive biophenols reduces the prevalence of Alzheimer's disease. Int J Mol Sci 2019;20. doi:10.3390/ijms20112797

16 Wade AT, Davis CR, Dyer KA, et al. A Mediterranean diet to improve cardiovascular and cognitive health: protocol for a randomised controlled intervention study. Nutrients 2017;9. doi:10.3390/ nu9020145

17 Aridi YS, Walker JL, Wright ORL. The association between the Mediterranean dietary pattern and cognitive health: a systematic review. Nutrients 2017;9. doi:10.3390/nu9070674

18 Anastasiou CA, Yannakoulia M, Kosmidis MH, et al. Mediterranean diet and cognitive health: initial results from the Hellenic longitudinal investigation of ageing and diet. PLoS One 2017;12:e0182048.

19 Yannakoulia M, Kontogianni M, Scarmeas N. Cognitive health and Mediterranean diet: just diet or lifestyle pattern? Ageing Res Rev 2015;20:74-8.

20 Feart C, Samieri C, Barberger-Gateau P. Mediterranean diet and cognitive health: an update of available knowledge. Curr Opin Clin Nutr Metab Care 2015;18:51-62.

21 Morris MC. Nutrition and risk of dementia: overview and methodological issues. Ann N Y Acad Sci 2016;1367:31-7.

22 Taylor MK, Swerdlow RH, Sullivan DK. Dietary Neuroketotherapeutics for Alzheimer's Disease: An Evidence Update and the Potential Role for Diet Quality. Nutrients 2019;11:1910.

23 Garcia-Penas JJ. [Epilepsy, cognition and ketogenic diet]. Rev Neurol 2018;66:S71-5.

24 Brandt J, Buchholz A, Henry-Barron B, et al. Preliminary report on the feasibility and efficacy of the modified Atkins diet for treatment of mild cognitive impairment and early Alzheimer's disease. $J$ Alzheimers Dis 2019;68:969-81.

25 Pistollato F, Iglesias RC, Ruiz R, et al. Nutritional patterns associated with the maintenance of neurocognitive functions and the risk of dementia and Alzheimer's disease: a focus on human studies. Pharmacol Res 2018;131:32-43.

26 Luchsinger JA, Tang M-X, Shea S, et al. Caloric intake and the risk of Alzheimer disease. Arch Neurol 2002;59:1258-63.

27 Martin CK, Anton SD, Han H, et al. Examination of cognitive function during six months of calorie restriction: results of a randomized controlled trial. Rejuvenation Res 2007;10:179-90.

28 Witte AV, Fobker M, Gellner R, et al. Caloric restriction improves memory in elderly humans. Proc Natl Acad Sci U S A 2009;106:1255-60.

29 Higgins JPT, Altman DG, Gøtzsche PC, et al. The Cochrane collaboration's tool for assessing risk of bias in randomised trials. BMJ 2011;343:d5928.

30 Higgins JP, Whitehead A. Borrowing strength from external trials in a meta-analysis. Stat Med 1996;15:2733-49.

31 Lu G, Ades AE. Combination of direct and indirect evidence in mixed treatment comparisons. Stat Med 2004;23:3105-24.

32 Bounthavong M, Zargarzadeh A, Hsu DI, et al. Cost-Effectiveness analysis of linezolid, daptomycin, and vancomycin in methicillinresistant Staphylococcus aureus: complicated skin and skin structure infection using Bayesian methods for evidence synthesis. Value Health 2011;14:631-9.

33 Ades AE, Sculpher M, Sutton A, et al. Bayesian methods for evidence synthesis in cost-effectiveness analysis. Pharmacoeconomics 2006;24:1-19.

34 Krahn U, Binder $\mathrm{H}$, König J. A graphical tool for locating inconsistency in network meta-analyses. BMC Med Res Methodol 2013;13:35.

35 Schwingshackl L, Chaimani A, Hoffmann G, et al. Impact of different dietary approaches on blood pressure in hypertensive and prehypertensive patients: protocol for a systematic review and network meta-analysis. BMJ Open 2017;7:e014736.

36 Salanti G, Ades AE, loannidis JPA. Graphical methods and numerical summaries for presenting results from multipletreatment meta-analysis: an overview and tutorial. $J$ Clin Epidemiol 2011;64:163-71.

37 Crainiceanu CM, Goldsmith AJ. Bayesian functional data analysis using WinBUGS. J Stat Softw 2010;32.

38 Dias S, Welton NJ, Sutton AJ, et al. Nice dsu technical support document 2: a generalised linear modelling framework for pairwise and network meta-analysis of randomised controlled trials. London, 2014.

39 Dias S, Welton NJ, Sutton AJ, et al. Nice dsu technical support document 4: inconsistency in networks of evidence based on randomised controlled trials. London, 2014. 
40 Salanti G, Del Giovane C, Chaimani A, et al. Evaluating the quality of evidence from a network meta-analysis. PLoS One 2014;9:e99682.

41 Higgins JPT, Jackson D, Barrett JK, et al. Consistency and inconsistency in network meta-analysis: concepts and models for multi-arm studies. Res Synth Methods 2012;3:98-110.

42 Jackson D, Barrett JK, Rice S, et al. A design-by-treatment interaction model for network meta-analysis with random inconsistency effects. Stat Med 2014;33:3639-54.

43 Bucher HC, Guyatt GH, Griffith LE, et al. The results of direct and indirect treatment comparisons in meta-analysis of randomized controlled trials. J Clin Epidemiol 1997;50:683-91.

44 Dias S, Welton NJ, Caldwell DM, et al. Checking consistency in mixed treatment comparison meta-analysis. Stat Med 2010;29:932-44.
45 Yang M, Yu Y, Lei P-G, et al. Comparative efficacy and safety of probiotics for the treatment of irritable bowel syndrome: a systematic review and network meta-analysis protocol. BMJ Open 2019;9:e027376.

46 Chiavaroli L, Kendall CWC, Braunstein CR, et al. Effect of pasta in the context of low-glycaemic index dietary patterns on body weight and markers of adiposity: a systematic review and metaanalysis of randomised controlled trials in adults. BMJ Open 2018;8:e019438.

47 Schwingshackl L, Knüppel S, Schwedhelm C, et al. Perspective: NutriGrade: a scoring system to assess and judge the MetaEvidence of randomized controlled trials and cohort studies in nutrition research. Adv Nutr 2016;7:994-1004. 